\title{
Pectus excavatum y carinatum en el síndrome de Marfan y síndromes similares: prevalencia e impacto clínico pulmonar y cardiovascular
}

\author{
María Elena Soto, ${ }^{1}$ Roberto Cano, ${ }^{2}$ C. Sergio Criales, ${ }^{3}$ Leonel Avendaño, ${ }^{4}$ Nilda Espínola 4 y \\ Carlos García ${ }^{5}$ \\ ${ }^{1}$ Departamento de Inmunología "Instituto Nacional de Cardiología "Ignacio Chávez"; ${ }^{2}$ Departamento de Resonancia Magnética "Instituto Nacional \\ de Cardiología "Ignacio Chávez"; ${ }^{3}$ Departamento de Tomografía Computada "Instituto Nacional de Cardiología "Ignacio Chávez"; ${ }^{4}$ Departamento \\ de Medicina Nuclear. "Instituto Nacional de Cardiología "Ignacio Chávez"; ${ }^{5}$ Departamento de Cirugía de Tórax "Hospital Infantil Privado". Ciudad \\ de México, México.
}

\section{Resumen}

Introducción: El pectus excavatum (PE) y el pectus carinatum (PC) son frecuentes en el síndrome de Marfan (SM) y en síndromes similares (SS). Los pacientes pueden evolucionar sin síntomas. En algunos hay depresión, trastornos de adaptación social, síntomas pulmonares y cardiovasculares, en los cuales hay controversia de su relación con el daño estructural del tórax. Objetivo: Evaluar la prevalencia del tipo de deformidad torácica en pacientes con SM y SS en una cohorte histórica y analizar el impacto clínico, pulmonar y cardiovascular. Método: Estudio prospectivo. Se incluyeron sujetos con criterios de Ghent y características específicas de cada síndrome, con expediente completo, ecocardiograma o resonancia magnética y tomografía computada, y pruebas de función respiratoria. Resultados: De un total de 338 pacientes con SM y SS, se detectaron 112 casos con deformidad torácica. Prevalencia de PE y PC en SM: 13.6 y 12.4; fue menor en SS. Hay compresión y desplazamiento de pulmón y cavidades cardiacas derechas por PE. Hay correlación entre el Índice de Haller y la presión sistólica de la arteria pulmonar incrementada es de $44(p=0.009)$. Conclusiones: La prevalencia de PE y PC en el SM y SS es alta, lo cual impacta en la función pulmonar y cardiovascular, en esas condiciones se requiere del manejo correctivo de la deformidad torácica y el objetivo no es estético.

PALABRAS ClAVE: Pectus excavatum. Pectus carinatum. Prevalencia. Daño pulmonar. Impacto cardiovascular.

\section{Abstract}

Introduction: Pectus excavatum (PE) and carinatum (PC) are common in Marfan syndrome (SM) and similar syndromes (SS). Patients can evolve without symptoms. In some there is depression, social adjustment disorders, pulmonary and cardiovascular symptoms in which there is controversy about their relationship with the structural damage of the thorax. Objective: To assess the prevalence of the type of thoracic deformity in patients with MS and SS in a historical and current cohort and to analyze the clinical, pulmonary and cardiovascular impact. Method: Prospective study. Subjects who met the Ghent criteria and who had a complete clinical record, an echocardiogram and/or magnetic resonance imaging, computed tomography and respiratory function tests were included. Results: Of a total of 338 patients with MS and SS, 112 cases with thoracic deformity were detected, the prevalence of PE and PC in SM 13.6 and 12.4, respectively, was lower in SS. There is compression and displacement of lung and right cardiac cavities by PE and the correlation between the Haller Index and the increased PASP is 44 ( $p=0.009)$. Conclusions: The prevalence of PE and PC in SM and SS is high, which impacts on lung function and cardiovascular damage, requires corrective management of the thoracic deformity and not only implies for aesthetic purposes.

KEY WORDS: Pectus excavatum. Pectus carinatum. Prevalence. Lung damage. Cardiovascular impact.

Correspondencia:

María Elena Soto

E-mail: mesoto50@ hotmail.com
Fecha de recepción: 16-08-2018

Fecha de aceptación: 20-08-2018 DOI: 10.24875/GMM. 18004581
Gaceta Med Mex. 2018;154(Suppl 2): S67-S78

Disponible en PubMed www.gacetamedicademexico.com 


\section{Introducción}

Las enfermedades del tejido conectivo conforman un grupo de padecimientos con diversa expresión clínica; la mejor conocida es el síndrome de Marfan (SM), pero existen otros padecimientos similares, con un fenotipo muy parecido y con características clínicas diferentes. ${ }^{1}$

En el SM, la incidencia varía de 1/5000 a 1-3/10 000, y se presenta en ambos sexos. ${ }^{2}$ En padecimientos similares, la incidencia no se conoce con exactitud.

En estos padecimientos, las diversas manifestaciones clínicas se han asociado con mutaciones del gen FBN-1, el cual se encuentra en el cromosoma 15q21.1. Este gen está compuesto de 65 exones y codifica la fibrilina-1, una glucoproteína con peso molecular de aproximadamente $350 \mathrm{kDa}$, componente principal de las microfibrillas de la matriz extracelular, lo que implica que las diversas mutaciones conllevan un daño en el tejido conectivo. ${ }^{3}$

Los padecimientos similares al SM pueden estar asociados también a mutaciones en el gen FBN-1, pero en ellos se sabe de la participación de mutaciones en los genes del factor de crecimiento transformante 1 y $2 .^{4}$

El diagnóstico es clínico. La nosología de Ghent permite identificarlos y clasificarlos, así como sospechar por características clínicas específicas la posibilidad de otros síndromes. Sin embargo, esto implica un reto en la identificación temprana del diagnóstico, lo cual se debe a que hay una amplia diversidad fenotípica, inclusive intrafamiliar, además de que genéticamente se ha atribuido a que existe un alto grado de mutaciones espontáneas, ${ }^{5,6}$ lo cual hace que la expresión clínica sea muy diferente.

Una de las características clínicas que sobresalen en estos pacientes son los defectos faciales y musculoesqueléticos, lo que ayuda a que sean identificables. La talla alta y los defectos del esternón, de las costillas y de la columna vertebral se presentan en la mayoría desde el nacimiento.

El pecho hundido o pectus excavatum (PE) es una de las deformidades más comunes, cuya prevalencia es del 0.1 al $2.6 \%$, ${ }^{7}$ seguido del pectus carinatum (PC) o «pecho de paloma» con un $0.6 \%{ }^{8,9} \mathrm{EI} \mathrm{PE}$ consiste en un sobrecrecimiento de la región condral junto con un desplazamiento de la parte inferior del esternón, lo que resulta en una deformidad cóncava ${ }^{10}$ que puede desencadenar fatiga, molestia o dolor torácico y compromete la respiración durante el ejercicio."11
Ocurre en 1 de 300-1000 nacidos vivos, la relación hombre: mujer es de 4:1 y su progresión es evidente durante la adolescencia. ${ }^{12}$

Estas formas de deformidad torácica están relacionadas con el crecimiento anormal de los cartílagos costocondrales. Se han identificado siete tipos de PE y pueden estar presentes en 27 distintos padecimientos genéticos humanos asociados con mutaciones en diferentes exones del gen FBN-1. ${ }^{13}$

La etiología de estas deformidades en modelos animales se ha encontrado asociada a mutaciones genéticas del gen de la sulfotransferasa 4 (GAL3ST4) y afecta la codificación y función de la proteína, lo que implica un desequilibrio del metabolismo de sulfato que puede ser la causa común de varias enfermedades congénitas con deformidades esqueléticas. Por otro lado, en estos pacientes la presencia de escoliosis asociada al PE puede ser común. ${ }^{14}$

También el gen GPR126 está implicado en los humanos, ${ }^{15-19}$ ya que actúa en el cartílago axial para regular la columna vertebral normal y el desarrollo del esternón en el ratón. El gen GPR126 parece dirigir la morfología esquelética normal regulando la morfogénesis del anillo fibroso, la supervivencia de los condrocitos y la expresión de Gal3st4 en tejidos de cartílago, lo cual ha sido demostrado en un modelo animal y proporciona datos robustos genéticos para determinar la fisiopatología de las deformidades del PE y de la enfermedad de columna idiopática..$^{20} \mathrm{De}$ forma habitual vemos que la deformidad torácica va asociada a una grave deformidad en la columna vertebral (Figura 1).

Por lo tanto, no puede ser considerado como una condición benigna, ya que en el PE puede haber compresión de cavidades cardiacas cuando hay disminución considerable del espacio entre el esternón y la columna vertebral, ${ }^{21,22}$ dando como consecuencia potenciales complicaciones hemodinámicas, exagerada interdependencia ventricular derecha y efectos en el crecimiento pulmonar y en la función de la vía aérea. ${ }^{8}$ Los síntomas como hipotensión ortostática se relacionan con una reducción del retorno venoso en un ventrículo izquierdo comprometido, lo cual es evaluado en los cambios posturales del paciente..$^{23}$

La decisión de realizar la corrección de ambos defectos ha sido controversial, debido a que hay estudios que informan de que la sobrevida en algunos es larga, aunque en otros se desconoce con certeza la repercusión clínica de estas deformidades. ${ }^{24}$

Estudios recientes han demostrado que el PE conduce a problemas cardiacos y pulmonares, y la 

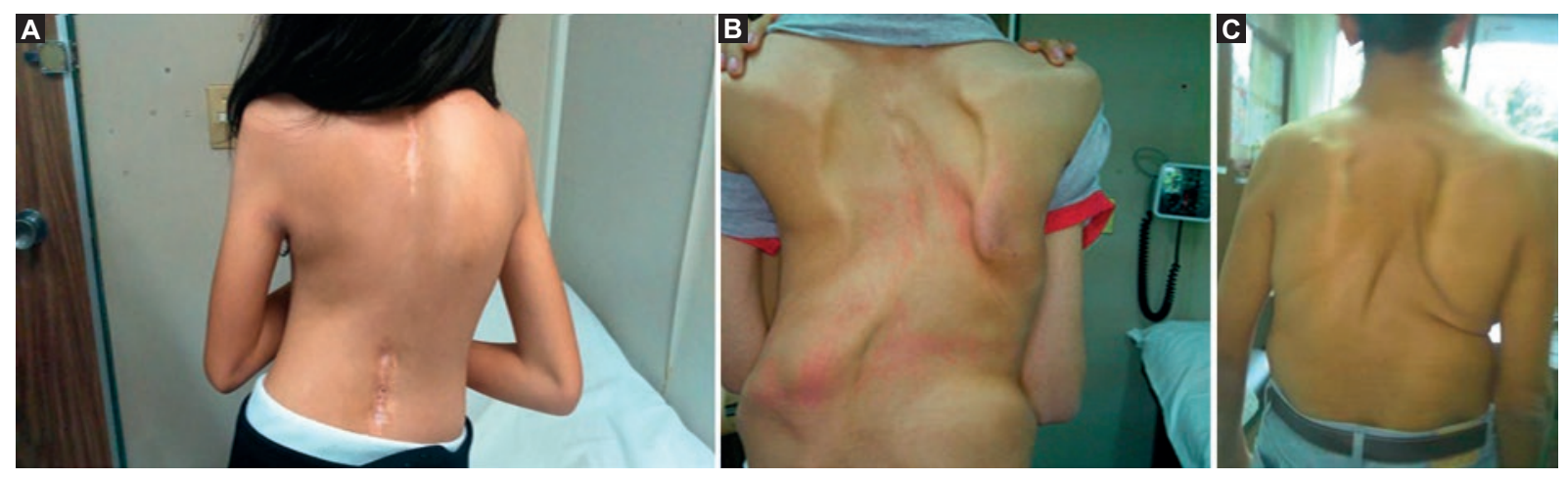

Figura 1. A: mujer de 15 años con síndrome de LD con PC y escoliosis grave, que ameritó durante su evolución tres intervenciones quirúrgicas de columna. B: hombre de 21 años con Shprintzen-Goldberg con PC que mantiene mejoría con uso de corsé. C: hombre de 38 años con SM, escoliosis y PE con índice de Haller de 4.5.

función del ventrículo izquierdo puede verse afectada. ${ }^{25}$ En sujetos con PE, el tratamiento debe ser analizado más allá de la estética, ${ }^{25}$ debido a que pueden cursar con afección psicológica, daño en la autoestima y tendencia al aislamiento social, lo cual hace que, en casos extremos, algunos adolescentes pueden desarrollar ideas suicidas. ${ }^{26}$ Dependiendo de la gravedad de la deformidad musculoesquelética, los pacientes refieren dolor paraesternal, disnea con esfuerzo y arritmias, que puede ser corregido con cirugía.

El instituto es un lugar de referencia para el tratamiento de la dilatación y la disección aórtica, una complicación catastrófica más temida; sin embargo, otros problemas médicos de igual significancia acompañan a estos pacientes, por lo que la investigación de cada uno de los problemas permitirá conocer mejor el trasfondo etiológico, al saber la frecuencia y el impacto de cada uno de ellos.

El objetivo de este estudio fue analizar la prevalencia de las deformidades torácicas en el SM y en síndromes similares (SS), y el impacto clínico pulmonar y cardiovascular.

\section{Método}

\section{Población}

Cohorte histórica de 338 casos con SM entre septiembre de 1982 y diciembre de 2017. Un total de 112 sujetos con deformidad torácica de todas las edades fueron incluidos; de ellos, 88 cumplieron con criterios de Ghent ${ }^{27}$ y correspondían a SM. Los pacientes con otros síndromes (Loeys-Dietz [LD], Beals Hecht, Shprintzen Goldberg, síndrome de MASS (de las siglas M (Mitral) A(Aortic) S (Skeletal) S (Skin) y enfermedad del tejido conectivo inespecífico [ETCI]) tuvieron las características clínicas que los distinguen. ${ }^{28-34}$ Se contó con el consentimiento informado.

La intervención correctiva de la deformidad por PE - ETCI se realizó en forma prospectiva, entre enero de 2011 y enero de 2018. Se evaluaron los pacientes que acudieron a la consulta externa del Instituto Nacional de Cardiología Ignacio Chávez. Se realizaron historia clínica completa, evaluación oftalmológica, evaluación cardiovascular y puntaje del score sistémico. Todos los pacientes tuvieron evaluación por ecocardiograma, resonancia magnética (RM) o tomografía computada (TC). A los casos detectados con compresión torácica e índice de Haller > 3.2 se les solicitaron pruebas respiratorias, espirometría y pletismografía. La mediciones del índice de Haller se evaluaron por un cardiólogo experto. A los sujetos detectados con compromiso pulmonar o cardiaco importante se les propuso intervención mediante la instalación de barras de Nuss. Los pacientes con dilatación aórtica en riesgo fueron intervenidos electivamente y en otros se realizó cirugía cardiaca de urgencia por disección aórtica.

\section{Ecocardiografía}

El cardiólogo-ecocardiografista realizó el ecocardiograma transtorácico con el paciente en decúbito lateral izquierdo. Se utilizó un equipo de ultrasonido IE35 (Philips Medical Systems, Bothell, Washington, EE.UU.) provisto de un transductor S5-1, con modo M, bidimensional y Doppler color, pulsado y continuo.

Se utilizaron los planos paraesternales en eje corto y largo, apical de cuatro, tres y dos cámaras, y supraesternal. En el eje largo paraesternal se valoró la presencia de prolapso de la válvula mitral y aórtica, y en el plano apical de cuatro cámaras se valoró la 
presencia de prolapso de la válvula tricúspide. La gravedad de la insuficiencia mitral, aórtica y tricuspídea se valoró con Doppler color y continuo, de acuerdo con los lineamientos descritos. La función sistólica del ventrículo izquierdo se evaluó en los planos apicales de cuatro y dos cámaras, calculando la fracción de expulsión del ventrículo izquierdo mediante el método de Simpson modificado, y se consideró normal cuando fue $>53 \% .{ }^{35}$ La presión sistólica de la arteria pulmonar (PSAP) se determinó utilizando la velocidad pico de la regurgitación tricuspídea en el plano apical de cuatro cámaras con Doppler color y continuo. Se calculó el gradiente transtricuspídeo mediante la ecuación de Bernoulli modificada, y a este se sumó la presión auricular derecha de acuerdo con el colapso de la vena cava inferior. La PSAP fue clasificada como leve (30-49 $\mathrm{mmHg})$, moderada (50-69 $\mathrm{mmHg}$ ) o grave (> $70 \mathrm{mmHg}$ ). Esta clasificación de hipertensión pulmonar se basó en el consenso de expertos del American College of Chest Physicians/American Thoracic Society. ${ }^{36}$

\section{Tomografía computada}

Se empleo un tomógrafo de 256 cortes y doble fuente de energía (SOMATOM Definition Flash; Siemens Healthcare, Forchheim, Germany). Se hicieron estudios y una colimación de $128 \times 0.6 \mathrm{~mm}$. Se empleó un protocolo de reducción de dosis de radiación con modulación de corriente del tubo basada en el eje z.

\section{Resonancia magnética}

Los estudios fueron realizados en un equipo SIEMENS Magnetom Avanto de 1.5 Teslas. Las secuencias para la visualización y la valoración del tórax son secuencias T1 (sangre negra) en corte axial a un grosor de corte de $8 \mathrm{~mm}$ abarcando la totalidad del tórax. La disminución del diámetro anteroposterior se evalúa a través del índice de Haller, el cual se obtiene dividiendo el diámetro transverso máximo del tórax entre el diámetro anteroposterior mínimo (medición que se realiza desde el borde anterior del cuerpo vertebral hasta el borde posterior del esternón). ${ }^{37}$

\section{Análisis estadístico}

Las variables con distribución gaussiana se expresaron con medidas de tendencia central, media y desviación estándar; las variables sin distribución normal,

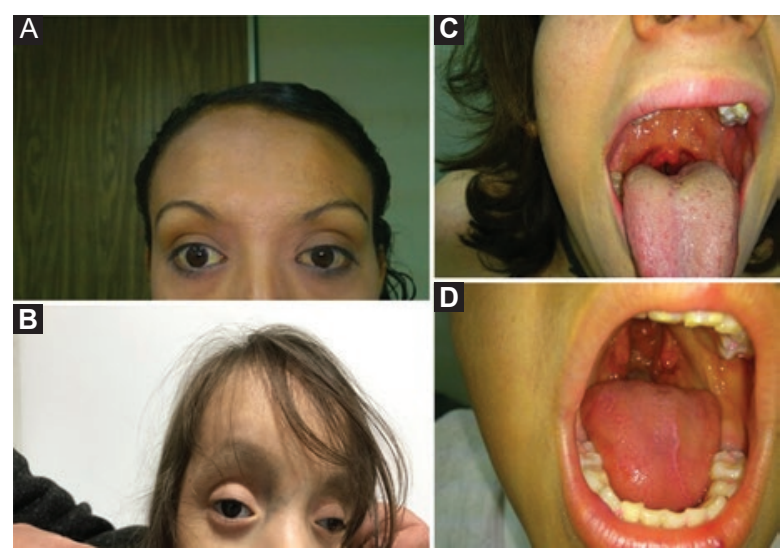

Figura 2. A: mujer de 20 años con $L D$ e hipertelorismo que fue intervenida por dilatación aortica de forma electiva con cirugía de David. B: niña de 3 años con $L D$, hipertelorismo y craneosinostosis. C: úvula bífida en un joven de 15 años con LD. D: úvula bífida con presentación diferente en un niño de 10 años con LD.

con mediana y valor mínimo y máximo; y las variables categóricas, con porcentajes. La comparación de medias con distribución normal se realizó con la prueba t de Student o U de Mann-Whitney; para variables categóricas, con prueba de ji al cuadrada con corrección de Yates, o según el caso del número esperado menor de 5 prueba exacta de Fisher. Los cálculos de prevalencia se hicieron con tablas de $2 \times 2$, considerando la población en riesgo y el tiempo del estudio.

\section{Resultados}

De un total de 338 pacientes con SM y SS se detectaron 112 casos con deformidad torácica, de los cuales 48 (43\%) eran mujeres y $64(57 \%)$ eran hombres, con una edad promedio de $28 \pm 14$ años. Los síndromes fueron SM en 88 (79\%), LD en 14 (13\%), Beals-Hecht en 3 (3\%), enfermedad del tejido conectivo inespecífico $(\mathrm{ETCl})$ en $3(3 \%)$ y síndrome de MASS en 2 (2\%). Las características demográficas de los síndromes de muestran en la Tabla 1.

La frecuencia de los criterios de Ghent en cada síndrome y las características que los distinguen se muestran en la Tabla 2. El hipertelorismo estuvo presente en 12 de 14 (86\%) de los casos con LD y en otros había úvula bífida (Figura 2).

En 112 casos, la frecuencia de PE fue de 57 (51\%,) y la de PC fue de 55 (49\%), de los cuales la deformidad torácica fue simétrica o asimétrica en 36 (32\%) y $21(18 \%)$ vs. 26 (23\%) y 29 (26\%), respectivamente. Del total de PE y PC, hubo formas graves de ambos (Figura 3), las cuales fueron encontradas en un total de $5(9 \%)$ y $7(16 \%)$ casos, respectivamente. 
Soto ME, et al.: Pectus excavatum y carinatum en el síndrome de Marfan y padecimientos similares

Tabla 1. Características demográficas de los pacientes con síndrome de Marfan y padecimientos similares

\begin{tabular}{|c|c|c|c|c|c|c|c|}
\hline & $\begin{array}{c}\text { SM } \\
\mathrm{n}=88(100)\end{array}$ & $\begin{array}{c}\text { LD } \\
\mathrm{n}=14(100)\end{array}$ & $\begin{array}{c}\text { ETCl } \\
\mathrm{n}=3(100)\end{array}$ & $\begin{array}{c}S G \\
n=2(100)\end{array}$ & $\begin{array}{c}\text { BH } \\
n=3(100)\end{array}$ & $\begin{array}{c}\text { MASS } \\
\mathrm{n}=2(100)\end{array}$ & Total \\
\hline Adultos & $80(90)$ & $7(50)$ & $1(34)$ & & $3(100)$ & $2(100)$ & 94 \\
\hline Niños & $8(10)$ & $7(50)$ & $2(66)$ & $2(100)$ & & & 18 \\
\hline Edad adultos & $27(5-64)$ & $32(21-38)$ & 22 & & $59(21-61)$ & $19(18-20)$ & 94 \\
\hline Edad niños & $13(9-16)$ & $10(5-32)$ & $14(13-15)$ & $12(7-16)$ & & & 18 \\
\hline Mujeres & $40(46)$ & $8(57)$ & 0 & 0 & 0 & 0 & 48 \\
\hline Hombres & $48(54)$ & $6(43)$ & $3(100)$ & $2(100)$ & $3(100)$ & $2(100)$ & 64 \\
\hline IMC & $29 \pm 13$ & $22 \pm 11$ & $16 \pm 4$ & $12 \pm 6$ & $28 \pm 2$ & $19 \pm 2$ & $17 \pm 10$ \\
\hline \multicolumn{8}{|l|}{ Lugar de origen. } \\
\hline CDMX & $43(38)$ & $6(5.3)$ & $3(2.7)$ & $1(0.9)$ & 0 & $2(1.7)$ & $55(49)$ \\
\hline Estado de México & $12(10.5)$ & $4(3.5)$ & 0 & 0 & 0 & 0 & $16(14)$ \\
\hline Puebla & $5(4.5)$ & 0 & 0 & $1(0.5)$ & 0 & 0 & $6(5.3)$ \\
\hline Guanajuato & $5(4.5)$ & $1(0.9)$ & 0 & 0 & 0 & 0 & $6(5.3)$ \\
\hline Veracruz & $3(2.7)$ & 0 & 0 & 0 & $1(0.8)$ & 0 & $4(3.5)$ \\
\hline Oaxaca & $3(2.7)$ & $1(0.8)$ & 0 & 0 & 0 & 0 & $4(3.5)$ \\
\hline Chiapas & $3(2.7)$ & 0 & 0 & 0 & 0 & 0 & $3(2.7)$ \\
\hline Michoacán & $2(1.8)$ & $1(0.9)$ & 0 & 0 & 0 & 0 & $3(2.7)$ \\
\hline Zacatecas & $2(1.8)$ & 0 & 0 & 0 & 0 & 0 & $2(1.8)$ \\
\hline Durango & $2(1.8)$ & 0 & 0 & 0 & 0 & 0 & $2(1.8)$ \\
\hline Sinaloa & $2(1.8)$ & 0 & 0 & 0 & 0 & 0 & $2(1.8)$ \\
\hline Tlaxcala & $2(1.8)$ & 0 & 0 & 0 & 0 & 0 & $2(1.8)$ \\
\hline Guerrero & $2(1.8)$ & 0 & 0 & 0 & 0 & 0 & $2(1.8)$ \\
\hline SLP & $1(0.9)$ & 0 & 0 & 0 & 0 & 0 & $1(0.9)$ \\
\hline BCS & $1(0.9)$ & 0 & 0 & 0 & 0 & 0 & $1(0.9)$ \\
\hline Tamaulipas & 0 & $1(0.9)$ & 0 & 0 & 0 & 0 & $1(0.9)$ \\
\hline Hidalgo & 0 & 0 & 0 & 0 & $1(0.9)$ & 0 & $1(0.9)$ \\
\hline Yucatán & 0 & 0 & 0 & 0 & $1(0.9)$ & 0 & $1(0.9)$ \\
\hline Total & $88(78.5)$ & $14(12.5)$ & $3(2.7)$ & 2 & 3 & 2 & 112 \\
\hline
\end{tabular}

BCS: Baja California Sur; BH: Beals Hecht; CDMX: Ciudad de México; ETCI: enfermedad del tejido conectivo inespecífico; IMC: índice de masa corporal; LD: Loeys-Dietz;

MASS: prolapso mitral, dilatación aórtica, alteraciones esqueléticas parecidas a Marfan y lesiones en piel; SG: Shprintzen Goldberg; SLP: San Luis Potosí; SM: síndrome de Marfan.

La relación del PE y la escoliosis se halló en 78 de 112 (70\%); el total de sujetos con escoliosis y PE fue de 39 de 57 (68\%), y con PC fue de 39 de 55 (71\%).

El promedio del índice de Haller en los pacientes con PE tuvo una mediana de 5.5 (rango: 2 a 53). La compresión de las cavidades derechas por PE ocurrió en 26 a de 57 (46\%), con una prevalencia del $7.6 \%$. El desplazamiento del corazón y el desplazamiento pulmonar se observó en 14 de 57 (25\%), con una prevalencia del $4 \%$.
El seguimiento prospectivo de los sujetos con PE después del año 2011 incluyó a 47 pacientes, de los cuales 10 fueron intervenidos quirúrgicamente por presentar dilatación o disección aórtica (ocho con cirugía de Bentall y dos con cirugía de David). La corrección del PE mediante barras de Nuss en los ocho primeros no se indicó por ser prioridad la intervención por dilatación o disección; los dos de cirugía electiva con procedimiento de David no tenían PE con efecto de compresión o desplazamiento de cavidades derechas. Los sujetos con 
Tabla 2. Distribución y frecuencia de los criterios de Ghent para la clasificación del síndrome de Marfan y signos clínicos que predominan en padecimientos similares

\begin{tabular}{|c|c|c|c|c|c|c|c|}
\hline Criterios de Ghent & $\begin{array}{c}\text { SM } \\
\mathrm{n}=88(100)\end{array}$ & $\begin{array}{c}\text { LD } \\
n=14(100)\end{array}$ & $\begin{array}{c}\text { ETCI } \\
\mathrm{n}=3(100)\end{array}$ & $\begin{array}{c}\text { SG } \\
n=2(100)\end{array}$ & $\begin{array}{c}\text { BH } \\
n=3(100)\end{array}$ & $\begin{array}{c}\text { MASS } \\
n=2(100)\end{array}$ & $\begin{array}{c}\text { Total } \\
112\end{array}$ \\
\hline $\mathrm{AHF}$ & $56(67)$ & $9(64)$ & 0 & $1(50)$ & $1(33)$ & 0 & $67(60)$ \\
\hline Luxación de cristalino & $46(53)$ & $2(14)$ & 0 & $2(100)$ & 0 & 0 & $50(45)$ \\
\hline Dilatación aórtica & $78(89)$ & $13(93)$ & 0 & $2(100)$ & $3(100)$ & $1(50)$ & $97(87)$ \\
\hline \multicolumn{8}{|l|}{ Puntaje sistémico } \\
\hline Steinberg/WM & $79(90)$ & $12(86)$ & $3(100)$ & $2(100)$ & $3(100)$ & $2(100)$ & $101(90)$ \\
\hline Pectus carinatum & $42(48)$ & $8(57)$ & $1(33)$ & $2(100)$ & $2(67)$ & 0 & $55(49)$ \\
\hline Pectus excavatum & $46(52)$ & $6(43)$ & $2(67)$ & 0 & $1(33)$ & $2(100)$ & \\
\hline Pie cavo & $52(59)$ & $13(93)$ & $3(100)$ & $2(100)$ & $2(67)$ & $1(50)$ & $73(65)$ \\
\hline \multicolumn{8}{|l|}{ Neumotórax } \\
\hline Ectasia dural & $68(77)$ & $9(64)$ & $3(100)$ & $2(100)$ & $2(67)$ & $1(50)$ & $86(77)$ \\
\hline Protrusión acetábulo & $7(8)$ & $1(7)$ & 0 & $1(50)$ & 0 & 0 & $9(8)$ \\
\hline Reducción SS/SI & $72(82)$ & $11(79)$ & $2(67)$ & $1(50)$ & $3(100)$ & $1(50)$ & $90(80)$ \\
\hline Escoliosis & $69(78)$ & $12(86)$ & $1(33)$ & $2(100)$ & $1(33)$ & $1(50)$ & $86(77)$ \\
\hline Extensión de codo R & $20(18)$ & $3(2.7)$ & 0 & $1(50)$ & $3(100)$ & 0 & $27(24)$ \\
\hline Rasgos faciales & $83(94)$ & $13(93)$ & $2(67)$ & $2(100)$ & $3(100)$ & $2(100)$ & $105(94)$ \\
\hline Miopía & $47(53)$ & $5(36)$ & 0 & $1(50)$ & $1(33)$ & 0 & $54(48)$ \\
\hline Estrías & $63(72)$ & $10(72)$ & $3(100)$ & $2(100)$ & $2(67)$ & $2(100)$ & $82(73)$ \\
\hline Prolapso valvular mitral & $61(69)$ & $9(64)$ & 0 & $2(100)$ & $1(33)$ & $2(100)$ & $75(67)$ \\
\hline Total PS & $11 \pm 3$ & $10 \pm 2$ & $7 \pm 1$ & $14 \pm 2$ & $9 \pm 1$ & $12 \pm 3$ & $11 \pm 3$ \\
\hline \multicolumn{8}{|l|}{ Características en SS } \\
\hline Hipertelorismo & 0 & $12(86)$ & 0 & $1(33)$ & 0 & 0 & $13(12)$ \\
\hline Úvula bífida & 0 & $8(57)$ & 0 & 0 & 0 & 0 & $8(7)$ \\
\hline Millia & 0 & $8(57)$ & 0 & 0 & 0 & 0 & $8(7)$ \\
\hline Craneosinostosis & 0 & 0 & 0 & $1(50)$ & 0 & 0 & $1(0.8)$ \\
\hline Arterias tortuosas & 0 & $7(50)$ & 0 & $1(50)$ & 0 & 0 & $8(0.8)$ \\
\hline Orejas de duende & 0 & 0 & 0 & 0 & $2(67)$ & 0 & $3(2.7)$ \\
\hline Contractura flexión & 0 & 0 & 0 & 0 & $3(100)$ & 0 & $3(2.7)$ \\
\hline
\end{tabular}

AHF: Antecedentes heredo-famiiares; BCS: Baja California Sur; BH: Beals Hecht; CDMX: Ciudad de México; ETCl: enfermedad del tejido conectivo inespecífico; LD: Loeys-Dietz; MASS: prolapso mitral, dilatación aórtica, alteraciones esqueléticas parecidas a Marfan y lesiones en piel; PS: Puntaje de score; R: Reducido; SG: Shprintzen Goldberg; SLP: San Luis Potosí; SS/SI: Segmento superior/segmento inferior; SM: síndrome de Marfan; SS: síndromes similares; WM: Walker Murdock.

PC seguidos de 2011 a la fecha fueron 48 , de los cuales en 20 hubo dilatación o disección (o ambas) y la cirugía se realizó con procedimiento de Bentall y Bono (BB); en seis de manera electiva se realizó procedimiento de David. El comparativo del grupo de deformidad torácica en el que se realizaron más cirugías con dilatación aórtica importante fue el grupo de PC $(p=0.01)$.
Todos contaron con estudio ecocardiográfico, el cual mostró desde la primera evaluación dilatación o disección aórtica (Figura 4).

La prevalencia global de PE y PC, así como la razón de momios y el intervalo de confianza del $95 \%$ en cada uno de los padecimientos, se muestran en la Tabla 3. 

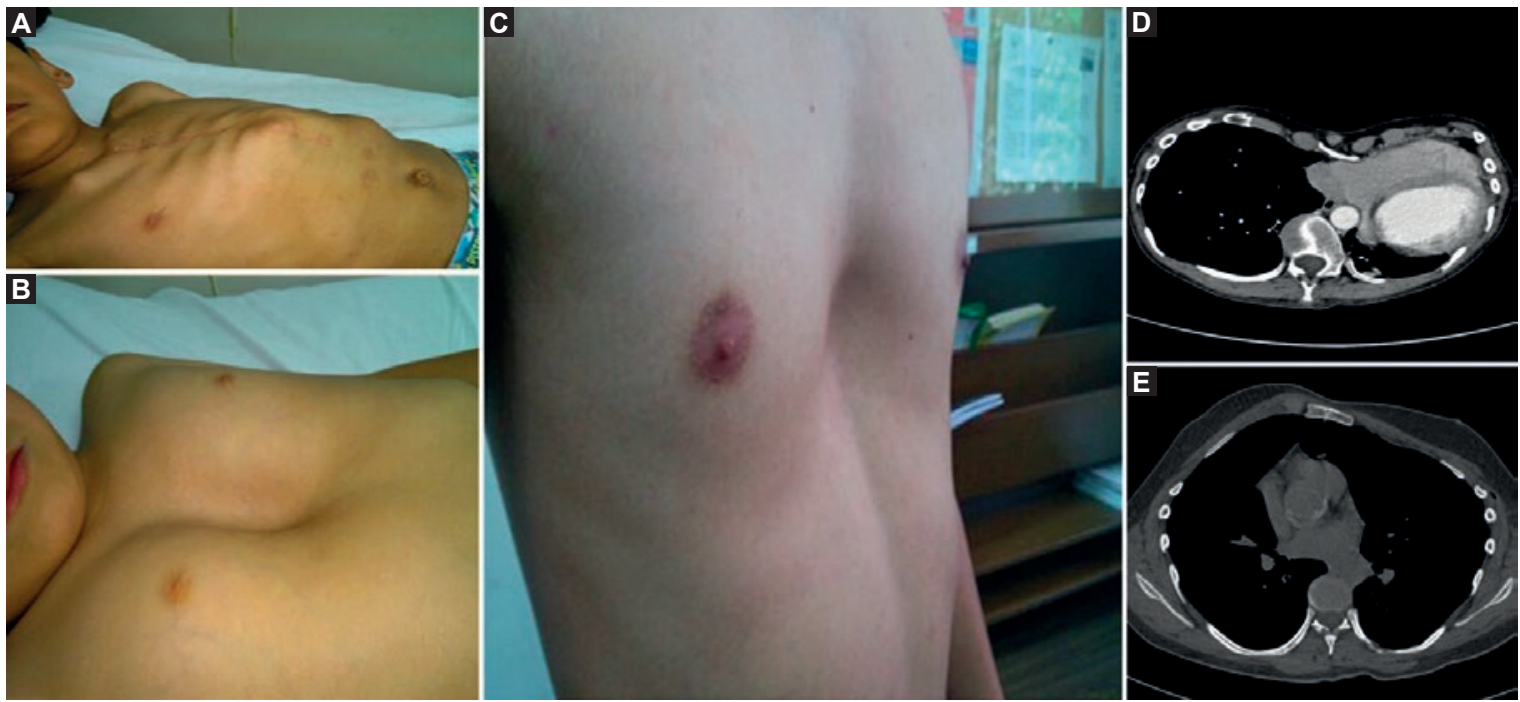

Figura 3. A: niño de 3 años con Shprintzen-Goldberg intervenido por dilatación aortica grave, el cual tiene PC grave. B: niño de 3 años con LD, PE e índice de Haller de 7.5. C: hombre de 32 años con PE e índice de Haller de 9.5.

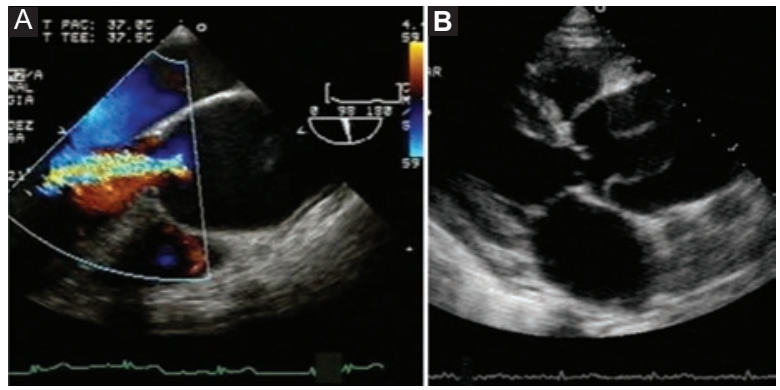

Figura 4. A: ecocardiograma transesofágico que muestra aneurisma de la aorta ascendente e insuficiencia aórtica moderada. B: eje largo paraesternal que muestra dilatación de aorta ascendente con colgajo de la íntima, que sugiere disección de la aorta ascendente.

\section{Enfermedad cardiovascular}

Se detectó dilatación aórtica en 97 (87\%), disección en 26 (23\%), prolapso valvular mitral (PVM) en $75(67 \%)$, prolapso valvular tricúspide en $22(20 \%)$ y prolapso valvular aórtico en $25(22 \%)$, insuficiencia mitral en 50 (45\%), insuficiencia aórtica en 49 (44\%) e insuficiencia tricúspide en 26 (23\%), y arritmias en $10(8 \%)$. La frecuencia de ellas por tipo de deformidad torácica se muestra en la Tabla 4.

De 14 pacientes que tuvieron desplazamiento de cavidades cardiacas por PE, 13 tuvieron PVM y un paciente tenía PC, lo que hace una frecuencia mayor de PVM en sujetos que presentan desplazamiento de cavidades por PE en la RM de 12 (intervalo de confianza del $95 \%$ : 2-126; $p=0.001$ ).

El promedio de la fracción de expulsión del ventrículo izquierdo fue de $55 \pm 10 \%$, y el de la PSAP fue de $29 \pm 10 \mathrm{mmHg}$.

\section{Enfermedad pullmonar}

Se encontró patrón restrictivo pulmonar en 25 (22\%), obstructivo en 4 (3.5\%), desplazamiento pulmonar en 15 (13\%), presencia de bullas en $10(9 \%)$, neumotórax en $3(2.5 \%)$ y oligohemia en $1(0.8 \%)$ (Figuras 5 y 6$)$. En los sujetos con PE, la correlación fue de 0.44; a mayor índice de Haller, mayor incremento de la PSAP, lo cual mostró significancia estadística ( $p=0.009$ ) (Figura 7). La comparación de estos hallazgos en relación con la deformidad torácica se muestra en la Tabla 4.

\section{Cirugías realizadas}

Con procedimiento de BB, un total de $30(27 \%)$, de las cuales 10 se realizaron entre 1982 y 2010 (periodo de 28 años) y 20 entre 2011 y 2017 (periodo de 6 años). Con procedimiento de David, 8 (7 \%) entre 2012 y 2017.

\section{Mortalidad}

Fallecieron cuatro pacientes, de los cuales dos tenían SM; a uno de ellos, de 38 años, se le realizó la primera cirugía de BB en 2003 y la segunda re-Bentall en 2015, y falleció por choque cardiogénico; el segundo paciente ingresó con disección aórtica Stanford A, con involucro del tronco braquiocefálico, carótida común, interna y externa derechas, y segmento proximal de ambas subclavias y carótida común izquierda, se le realizó cirugía de BB en 2015, y falleció en su casa por nueva disección aórtica abdominal. 
Tabla 3. Prevalencia general del pectus excavatum y del pectus carinatum en cada síndrome

\begin{tabular}{|c|c|c|c|c|}
\hline & \multicolumn{2}{|c|}{ Pectus excavatum } & \multicolumn{2}{|c|}{ Pectus carinatum } \\
\hline & Prevalencia & RM (IC 95\%) & Prevalencia & (IC 95\%) \\
\hline Marfan & 13.6 & $14(11.9-51.8)$ & 12.4 & $15(7.9-32.1)$ \\
\hline Loeys Dietz & 1.7 & $4(1.3-12.06)$ & 2.3 & $7.8(2.6-23.6)$ \\
\hline ETCl & 0.59 & $10(0.9-114)$ & 0.29 & $2.6(0.23-29.2)$ \\
\hline Beals Hecht & 0.29 & $2.4(0.22-27.9)$ & 0.59 & $10(0.94-119)$ \\
\hline Shprintzen Goldberg & 0 & - & 0.59 & - \\
\hline MASS & 0.59 & $1.1(0.23-23.5)$ & 0 & - \\
\hline
\end{tabular}

ETCI: enfermedad del tejido conectivo inespecífico; IC: intervalo de confianza; MASS: prolapso mitral, dilatación aórtica, alteraciones esqueléticas parecidas a Marfan y lesiones en piel; RM: razón de momios.
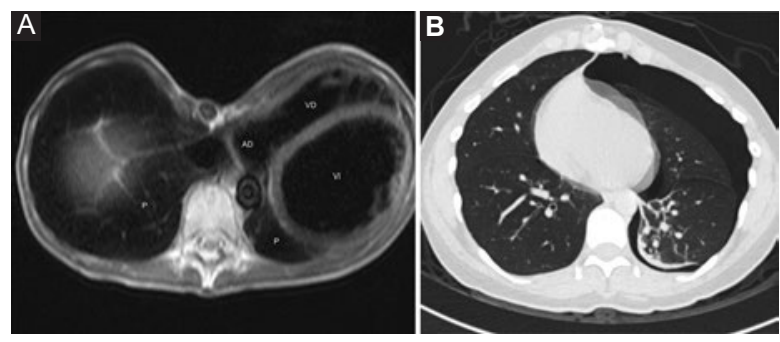

Figura 5. A: joven de 18 años con SM e índice de Haller de 18. Resonancia Magnética. Secuencia T1 en corte axial. Desplazamiento cardiaco a hemitórax izquierdo con compresión extrínseca de las cavidades derechas, reducción importante del diámetro entre el cuerpo vertebral y el esternón. B: joven de 17 años con $L D$ y PC, con extenso neumotórax izquierdo que desplaza al mediastino en sentido contralateral y que se asocia con adherencias pleurales hacia el lóbulo superior.
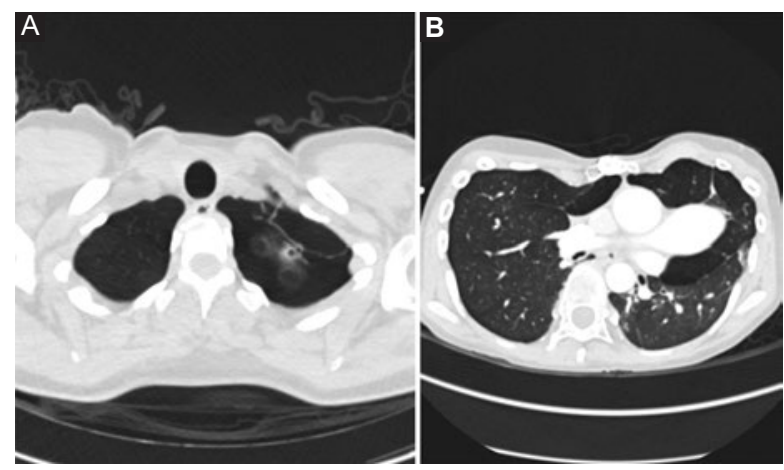

Figura 6. A: joven de 17 años con LD y bullas subpleurales. B: joven de 15 años con aneurisma del arco aórtico que se extiende desde el arco proximal hasta el istmo con un diámetro de $61 \mathrm{~mm}$. Muestra aéreas de oligohemia pulmonar.

De los otros dos fallecimientos, uno con síndrome de LD, de 29 años, tuvo disección Stanford A DeBakey III, insuficiencia aórtica grave y dilatación aórtica mayor de $90 \mathrm{~mm}$, fue intervenido de urgencia y falleció a los 4 días posteriores por choque cardiogénico, y el otro paciente, también de 29 años, con Shprintzen Goldberg, ingresó con insuficiencia aórtica

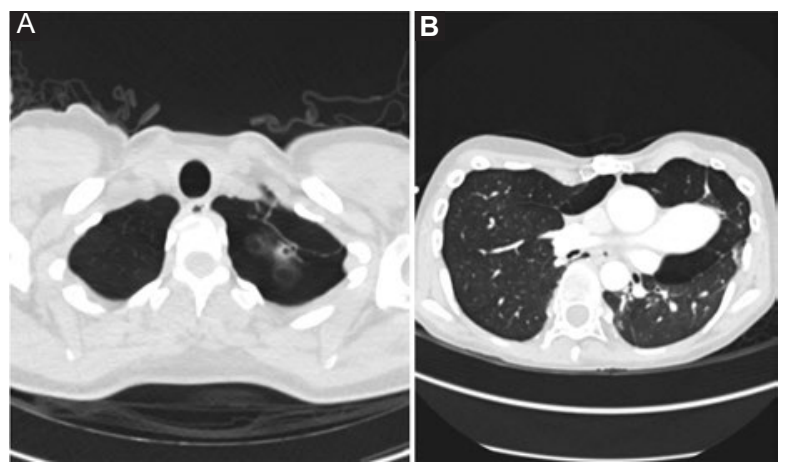

Figura 7. Imagen que muestra la correlación entre el índice de Haller y la PSAP.

importante y dilatación de senos de Valsalva de $98 \mathrm{~mm}$, y falleció durante su estancia y preparación quirúrgica por fibrilación ventricular e hipotensión con paro cardiorrespiratorio. Los tres intervenidos lo fueron mediante el procedimiento de BB.

Los hallazgos clínicos, pulmonares y cardiovasculares de acuerdo con el tipo de deformidad torácica se muestran en la Tabla 4.

De los 112 pacientes se pudo seguir prospectivamente para el estudio actual, a partir de 2011, a 104, ya los otros dejaron de venir entre 2006 y 2011, pero se localizaron por vía telefónica y estaban vivos.

De los pacientes con SM que presentaban compresión cardiaca de cavidades derechas y fueron intervenidos mediante instalación de barras de NUSS se muestran los datos clínicos en la Tabla 5.

\section{Limitación}

No se estudiaron de forma prospectiva aspectos psicosociales ni síntomas asociados a depresión. Los pacientes estudiados tenían en su mayoría larga evolución de la enfermedad, y se requiere un estudio en 
Soto ME, et al.: Pectus excavatum y carinatum en el síndrome de Marfan y padecimientos similares

Tabla 4. Frecuencias de hallazgos cardiovasculares y pulmonares de acuerdo con la deformidad torácica

\begin{tabular}{|c|c|c|c|c|}
\hline Complicación cardiovascular & Total $112(100 \%)$ & Pectus excavatum $\mathrm{n}=57(51 \%)$ & Pectus carinatum $n=55$ (49\%) & p \\
\hline Dilatación aórtica & $97(87)$ & $46(41)$ & $51(46)$ & 0.09 \\
\hline Disección aórtica & $26(23)$ & $9(8)$ & $17(15)$ & 0.09 \\
\hline Prolapso valvular mitral & $75(67)$ & $41(37)$ & $34(30)$ & NS \\
\hline Prolapso valvular tricúspide & $22(20)$ & $11(10)$ & $11(10)$ & NS \\
\hline Prolapso valvular aórtico & $25(22)$ & $10(9)$ & $15(13)$ & NS \\
\hline Insuficiencia mitral & $50(45)$ & $25(22)$ & $25(22)$ & NS \\
\hline Insuficiencia de tricúspide & $26(23)$ & $16(14)$ & $10(9)$ & NS \\
\hline Insuficiencia aórtica & $49(44)$ & $22(20)$ & $27(24)$ & NS \\
\hline \multicolumn{5}{|l|}{ Complicación pulmonar } \\
\hline Patrón restrictivo & $25(22)$ & $20(18)$ & $5(4)$ & 0.0001 \\
\hline Patrón obstructivo & $4(3.5)$ & $4(3.5)$ & 0 & NS \\
\hline Neumotórax & $3(2.5)$ & $2(1.7)$ & $1(0.8)$ & NS \\
\hline Bullas & $4(3.5)$ & $2(1.75)$ & $2(1.75)$ & NS \\
\hline Enfisema paraseptal & $1(0.8)$ & $1(0.8)$ & 0 & NS \\
\hline Oligohemia & $1(0.8)$ & $1(0.8)$ & 0 & NS \\
\hline Desplazamiento de cavidades & $15(13)$ & $14(12.5)$ & $1(0.5)$ & 0.0004 \\
\hline Compresión de cavidades derechas & $27(24)$ & $27(24)$ & 0 & 0.0001 \\
\hline
\end{tabular}

el que la evaluación basal de las pruebas respiratorias se realice para determinar si los cambios estructurales torácicos incrementan la condición o el daño cardiopulmonar.

\section{Discusión}

En este estudio, la prevalencia de la deformidad torácica en el SM y SS es mayor que lo informado en la literatura. ${ }^{7-9}$ La progresión de la deformidad torácica ocurre durante la adolescencia, ${ }^{38-42}$ y por lo tanto en esta etapa es cuando los síntomas y los aspectos psicosociales pueden ser más evidentes, y sin embargo pueden ser menos considerados por el paciente o por el médico. ${ }^{43}$

En el SM y SS la principal complicación catastrófica es la dilatación y la disección de aorta, la cual es progresiva y disminuye la sobrevida con una tasa alta de mortalidad. Sin embargo, no hay síntomas específicos cuando la dilatación progresa, y los que pueden presentarse son inusuales. Cuando ocurre la disección aórtica los pacientes presentan dolor abdominal agudo o dolor en el pecho y la espalda; no obstante, también puede presentarse atípicamente con un dolor mínimo o nulo, lo que dificulta el diagnóstico, pues puede tratarse de sintomatología puramente pulmonar relacionada con neumotórax espontáneos.

El seguimiento de las cirugías realizadas en pacientes con SM por disecciones combinadas de tipo $\mathrm{A}$ y $B$ muestra una tasa de supervivencia estimada a 5 años del $80,1 \%$ y una tasa de reintervención del $55,3 \%$; por lo tanto, una tasa tan alta de reintervenciones pone de relieve la necesidad de una vigilancia y un tratamiento cuidadosos para los pacientes con SM que sobreviven a una disección aguda. ${ }^{44}$

El punto principal en estos padecimientos es reconocer que la mayor causa de mortalidad está relacionada con el incremento del diámetro aórtico; sin embargo, hay daño sistémico y generalizado, por lo que un alto grado de conocimiento y sospecha para integrar la compleja problemática de estos síndromes lleva implícito que requieren un manejo multidisciplinario que incluya reumatólogo, oftalmólogo, cardiólogo, ortopedista, odontólogo, psicólogo y ginecólogo en los casos de embarazo, con un consejo genético oportuno, para ofrecer integralmente la atención que se merecen.

En esta serie se puede establecer que hay una gran relevancia en tomar en cuenta toda la información 
Tabla 5. Pacientes con síndrome de Marfan y pectus excavatum sometidos a cirugía correctiva del tórax

\begin{tabular}{|c|c|c|c|c|c|}
\hline Caso & $\begin{array}{l}\text { Año de } \\
\text { ingreso }\end{array}$ & Sexo & Edad & Descripción & Cirugía \\
\hline 1 & 2011 & $\mathrm{H}$ & 11 & $\begin{array}{l}\text { 10/09/2010 Inicio con precordial, cianosis } \\
\text { 26/05/2011 Acudió a valoración, el ECO mostró PVM y PVT } \\
\text { e IM leve. Notaron PE. } \\
\text { 09/08/2012 Diagnóstico de SM, la RM mostró PE, índice } \\
\text { de Haller de } 4.5 \text { que limitaba movimiento de cavidades } \\
\text { derechas en diástole. Las PR con patrón restrictivo. }\end{array}$ & $\begin{array}{l}\text { 19/02/2013 } \\
\text { Instalación de barras de Nuss. } \\
\text { 13/07/2016 } \\
\text { Retiro de barras de Nuss. }\end{array}$ \\
\hline 2 & 2001 & $\mathrm{H}$ & 32 & $\begin{array}{l}\text { 20/01/1986 El paciente a los } 5 \text { años con palpitaciones, fue } \\
\text { evaluado FINC, le indicaron verapamilo. } \\
\text { 12/03/2001 Continuo con palpitaciones y disnea, acudió } \\
\text { al INC, se diagnosticó SM, el ECO reportó IM, PVM, FA } \\
\text { paroxística, y la RM PE con índice de Haller de 5.6. } \\
\text { 20/03/2013 Incrementa la disnea, el ECO mostró IM grave y } \\
\text { la RM PE con índice de Haller de 9.5, el corazón y pulmón } \\
\text { desplazados sin dilatación aortica. Las PR con patrón } \\
\text { restrictivo. }\end{array}$ & $\begin{array}{l}\text { 20/03/2013 } \\
\text { Instalación de barras de Nuss. } \\
\text { 09/10/2014 } \\
\text { Cirugía para cambio valvular mitral. }\end{array}$ \\
\hline 3 & 2014 & $\mathrm{H}$ & 25 & $\begin{array}{l}\text { 13/01/2014 El paciente con dolor torácico y palpitaciones, } \\
\text { se diagnosticó SM. El ECO mostró aneurisma subvalvular } \\
\text { mitral, IM leve. } \\
\text { 18/02/2014 La RM PE con índice de Haller de } 5.6 \text {. } \\
\text { Compresión del tercio medio y apical de VD e IM. Las PR } \\
\text { normales. }\end{array}$ & $\begin{array}{l}\text { 01/12/2014 } \\
\text { Instalación de barras de Nuss. }\end{array}$ \\
\hline 4 & 2001 & $\mathrm{H}$ & 21 & $\begin{array}{l}\text { 13/01/2001 El padre del paciente con diagnóstico de SM } \\
\text { presentó disección aórtica y se revisaron los familiares. } \\
\text { 05/08/09 El hijo (caso) fue diagnosticado con SM. El ECO } \\
\text { mostro PVM, PVT y la RM PE con índice de Haller de } 10 \text { sin } \\
\text { restricción respiratoria. } \\
\text { 18/07/2012 Lumbalgia, dolor precordial, palpitaciones. } \\
\text { 12/01/2015 La RM mostró incremento del índice de Haller } \\
\text { a } 32 \text { y desplazamiento del corazón del lado Izquierdo con } \\
\text { dilatación aórtica. }\end{array}$ & $\begin{array}{l}\text { 22/01/2015 } \\
\text { Instalación de barras de NUSS. }\end{array}$ \\
\hline 5 & 2015 & $\mathrm{H}$ & 9 & $\begin{array}{l}\text { 9/12/2014 Dolor torácico ocasional y disnea. } \\
\text { 13/03/2015 Diagnóstico de SM, en un estudio de TC } \\
\text { se mostró PE con índice de Haller de } 5.9 \text { que restringe } \\
\text { el movimiento diastólico del VD en su tercio basal, sin } \\
\text { dilatación aórtica. PR con patrón restrictivo. }\end{array}$ & $\begin{array}{l}\text { 01/05/2015 } \\
\text { Instalación de barras de Nuss. }\end{array}$ \\
\hline 6 & 2016 & $\mathrm{H}$ & 20 & $\begin{array}{l}\text { 19/05/2016 Disnea ocasional y dolor torácico, problemas } \\
\text { psicosociales relacionados con el pectus. Se diagnosticó } \\
\text { ETCI. } \\
\text { 20/07/2016 El ECO mostró PVN, la RM PE con índice de } \\
\text { Haller de 4.2, sin dilatación aórtica. PR normales. }\end{array}$ & $\begin{array}{l}\text { 08/03/2017 } \\
\text { Instalación de barras de Nuss. }\end{array}$ \\
\hline 7 & 2017 & $\mathrm{H}$ & 16 & $\begin{array}{l}\text { 27/03/2017 El padre con SM falleció por disección aórtica. } \\
\text { Fue evaluada toda la familia y el paciente con SM. La } \\
\text { RM mostró PE con índice de Haller de 9, compresión de } \\
\text { la pared libre del ventrículo derecho, disfunción sistólica } \\
\text { ventricular y dilatación aórtica. }\end{array}$ & $\begin{array}{l}\text { 03/07/2017 } \\
\text { Instalación de barras de Nuss. }\end{array}$ \\
\hline
\end{tabular}

ECO: ecocardiograma; FINC: otro hospital; IM: insuficiencia mitral; INC: Instituto Nacional de Cardiología; PE: pectus excavatum; PR: pruebas respiratorias; PVM: prolapso valvular mitral; PVT: prolapso valvular tricúspide; RM: resonancia magnética; TC: tomografía computada.

pertinente, buscando pistas importantes con el fin de desarrollar un diagnóstico diferencial en casos de reto diagnóstico.

Las deformidades torácicas y la escoliosis son comunes en estos pacientes, y en esta serie se confirma que la escoliosis está altamente relacionada con la deformidad del tórax en el SM y los SS, lo cual tiene relación con disfunción pulmonar, que se expresa clínicamente en diferentes formas. Sin embargo, hay informes de que la condición de daño pulmonar asociada a esta relación de escoliosis con PE puede mejorar una vez que se realiza la corrección de la deformidad torácica, y hay además mejoría de la función ventricular derecha, pero aún es controversial. ${ }^{44}$ 
La intervención correctiva del tórax en esta serie tiene muy corta evolución; sin embargo, la mejoría clínica ha sido evidente, aunque se requiere seguimiento a largo plazo para determinar si la intervención mejora las condiciones pulmonares y cardiacas, con preguntas de investigación muy concretas.

Aunque los síntomas pulmonares no son una característica principal en el SM, es evidente lo encontrado en esta serie, que muchos de los pacientes tienen cierto grado de patología pulmonar subyacente. El neumotórax espontáneo unilateral o bilateral es una manifestación clínica común, y su prevalencia es del $4.4 \%$; en esta serie fue menor, pero es factible que algunos casos previos a la intervención realizada lo hayan presentado y en su momento no se realizaran los estudios pertinentes de imagen. ${ }^{45}$ Otras anomalías, como las congénitas lobulares, han sido reportadas, así como cambios fibróticos en panal de abeja y enfisema. ${ }^{46-49}$

En pacientes con $\mathrm{PE}$, algunas de las alteraciones cardiovasculares, como palpitaciones, dolor torácico ocasional y disnea, o bien esofágicas manifestadas por dolor torácico «atípico» o reflujo gastroesofágico, son el resultado de la compresión del mediastino causada por el choque del esternón, que es la causa de muchos de los síntomas y de los hallazgos electrocardiográficos. ${ }^{50}$

La compresión ventricular derecha ha sido detectada en estos pacientes con y sin desplazamiento de las estructuras cardiacas. Por ello se ha sugerido que la evaluación anatómica y funcional es de gran importancia para una comprensión integral de la enfermedad y un plan de evaluación de cambios en el seguimiento y tratamiento adecuados, en los que el apoyo de la imagen es fundamental. ${ }^{51} \mathrm{La}$ frecuencia y la prevalencia de esta condición se confirman en esta serie, y tiene relevancia clínica y funcional.

Los estudios de prevalencia indican al investigador la abundancia de los eventos que pueden presentarse en una población susceptible a ciertas complicaciones dentro de su enfermedad, y tiene su mayor utilidad en los estudios de planificación de servicios sanitarios. Las alteraciones musculoesqueléticas de estos pacientes deben ser tomadas en cuenta para su atención oportuna y pueden ser la base integral del estudio etiológico, ya que pueden guardar amplia interrelación con situaciones graves de la enfermedad.

El PE y el PC tienen alta prevalencia en el SM y el LD. EI PE es progresivo y conlleva la compresión de estructuras cardiacas y pulmonares, lo cual está relacionado con disfunción pulmonar. Un hallazgo relevante es que hay correlación entre un mayor índice de Haller y la PSAP, lo cual no ha sido previamente reportado y puede tener relevancia clínica pronóstica.

Las manifestaciones cardiovasculares parecen estar relacionadas con el desplazamiento y la compresión de las cavidades derechas. Sin embargo, a pesar del impacto que la deformación por PE tiene sobre el funcionamiento de los órganos en el tórax y ha sido informado desde hace más de dos décadas, ${ }^{52,53}$ se requieren estudios prospectivos proyectados para evaluar cada una de las manifestaciones, relacionadas con cada tipo de deformidad, con la presencia de prolapso valvular, arritmias y disfunción ventricular derecha.

Los métodos de imagen son complementarios entre sí y aportan gran conocimiento de los cambios vasculares y esqueléticos durante la evaluación de estos pacientes. La ecocardiografía podría determinar el estado basal al momento del diagnóstico y los cambios durante el efecto compresivo o no compresivo de las deformidades torácicas. La RM y la TC desempeñan un papel fundamental en los cambios estructurales para la decisión correctiva de la deformidad, principalmente en sujetos con disfunción pulmonar y cardiaca, cuya interrelación requiere una investigación precisa y enfocada a cada problema.

\section{Bibliografía}

1. Hoffjan S. Genetic dissection of Marfan syndrome and related connective tissue disorders: an update 2012. Mol Syndromol. 2012;3:47-58.

2. Judge DP, Dietz HC. Marfan' syndrome. Lancet. 2005;366:1965-76.

3. Collod-Béroud G, LeBourdelles S, Ades L, Ala-Kokko L, Booms P, BoxerM, etal. Update of the UMD-FBN1 mutation database and creation of an FBN1 polymorphism database. Int J ClinExp Med. 2015;8:7419-24.

4. Stheneur C, Collod-Béroud G, Faivre L, Gouya L, Sultan G, Le Parc JM, et al. Identification of 23 TGFBR2 and 6 TGFBR1 gene mutations and genotype-phenotype investigations in 457 patients with Marfan syndrome type I and II, Loeys-Dietz syndrome and related disorders. Hum Mutat. 2008;29:E284-95.

5. Campens L, Callewaert B, Muiño Mosquera L, Renard M, Symoens S, De Paepe A, et al. Gene panel sequencing in heritable thoracic aortic disorders and related entities results of comprehensive testing in a cohort of 264 patients. Orphanet J Rare Dis. 2015;10:9.

6. Liu DL, Cao JH, Yang J, He F, Wang Y, Fan N, et al. A novel mutation in fibrillin-1 gene identified in a Chinese family with Marfan syndrome. Int $\mathrm{J}$ Clin Exp Med. 2015;15:7419-24.

7. Cobben JM, Oostra RJ, van Dijk FS. Pectus excavatum and carinatum. Eur J Med Genet. 2014;57:414-7.

8. Coskun ZK, Turgut HB, Demirsoy S, Cansu A. The prevalence and effects of pectus excavatum and pectus carinatum on the respiratory function in children between 7-14 years old. Indian J Pediatr. 2010;77:1017-9.

9. Westphal FL, Lima LC, Lima Neto JC, Chaves AR, Santos Júnior VL, Ferreira BL. Prevalence of pectus carinatum and pectus excavatum in students in the city of Manaus, Brazil. J Bras Pneumol. 2009;35:221-6.

10. Kelly Jr RE. Pectus excavatum: historical background, clinical picture, preoperative evaluation and criteria for operation. Semin Pediatr Surg. 2008;17:181-93.

11. Lesbo M, Tang M, Nielsen HH, Frøkiær J, Lundorf E, Pilegaard HK, et al. Compromised cardiac function in exercising teenagers with pectus excavatum. Interact Cardiovasc Thorac Surg. 2011;13:377-80.

12. Williams AM, Crabbe DC. Pectus deformities of the anterior chest wall. Paediatr Respir Rev. 2003;4:237-42. 
13. Reyes-Hernández OD, Palacios-Reyes $C$, Chávez-Ocaña S, Cortés-Malagón EM, Alonso-Themann PG, Ramos-Cano V, et al. Skeletal manifestations of Marfan syndrome associated to heterozygous R2726WFBN1 variant: sibling case report and literature review. BMC Musculoskele Disord. 2016:17:79.

14. Hong JY, Suh SW, Park HJ, Kim YH, Park JH, Park SY. Correlations of adolescent idiopathic scoliosis (EI) and pectus excavatum. J Pediat Orthop. 2011:31:870-4.

15. Kou I, Takahashi Y, Johnson TA, Takahashi A, Guo L, Dai J, et al. Genetic variants in GPR126 are associated with adolescent idiopathic scoliosis. Nat Genet. 2013;45:676-9.

16. Sharma S, Gao X, Londono D, Devroy SE, Mauldin KN, Frankel JT, et al Genome-wide association studies of adolescent idiopathic scoliosis suggest candidate susceptibility genes. Hum Mol Genet. 2011;20:1456-66

17. Takahashi Y, Kou I, Takahashi A, Johnson TA, Kono K, Kawakami N, et al. A genome-wide association study identifies common variants near LBX1 associated with adolescent idiopathic scoliosis. Nat Genet. 2011:43:1237-40.

18. Wu S, Sun X, Zhu W, Huang Y, Mou L, Liu M, et al. Evidence for GAL3ST4 mutation as the potential cause of pectus excavatum. Cell Res. 2012;22:1712-5

19. Xu JF, Yang GH, Pan XH, Zhang SJ, Zhao C, Qiu BS, et al. Association of GPR126 gene polymorphism with adolescent idiopathic scoliosis in Chinese populations. Genomics. 2015;105:101-7.

20. Karner CM, Long F, Solnica-Krezel L, Monk KR, Gray RS. Gpr126/Adgrg6 deletion in cartilage models idiopathic scoliosis and pectus excavatum in mice. Hum Mol Genet. 2015;24:4365-73.

21. Kragten HA, Siebenga J, Höppener PF, Verburg R, Visker N. Symptomatic pectus excavatum in seniors (SPES): a cardiovascular problem? A prospective cardiological study of 42 seniors with a symptomatic pectus excavatum. Neth Heart. 2011;19:73-8.

22. Tardy MM, Filaire M, Patoir A, Gautier-Pignonblanc P, Galvaing G, Kwiatkowski $F$, et al. Exercise cardiac output limitation in pectus excavatum. J Am Coll Cardiol. 2015;66:976-7.

23. Bafus BT, Chiravuri D, van der Velde ME, Chu BI, Hirshl R, Farley FA Severe hypotension associated with the prone position in a child with scoliosis and pectus excavatum undergoing posterior spinal fusion. J Spinal Disord Tech. 2008;21:451-4

24. Kelly Jr RE, Lawson ML, Paidas CN, Hruban RH. Pectus excavatum in a 112-year autopsy series: anatomic findings and the effect on survival. J Pediatr Surg. 2005;40:1275-8.

25. Gürsu AH, Karagün BS, Korkmaz O, Gürsu SS, Uçar MA. Correlation between Haller index and echocardiographic and spirometric findings in children with pectus excavatum. Turk Kardiyol Dern Ars. 2014;42:259-64.

26. Ji Y, Liu W, Chen S, Xu B, Tang Y, Wang X, et al. Assessment of psychosocial functioning and its risk factors in children with pectus excavatum. Health Qual Life Outcomes. 2011:9:28.

27. Loeys BL, Dietz HC, Braverman AC, Callewaert BL, De Backer J, Devereux RB, et al. The revised Ghent nosology for the Marfan syndrome. J Med Genet. 2010;47:476-85.

28. Yetman AT, Beroukhim RS, Ivy DD, Manchester D. Importance of the clinical recognition of Loeys-Dietz syndrome in the neonatal period. $\mathrm{Pe}-$ diatrics. 2007;119:1199-202.

29. Johnson PT, Chen JK, Loeys BL, Dietz HC, Fishman EK. Loeys-Dietz syndrome: MDCT angiography findings. AJR Am J Roentgenol. 2007;189:W29-35.

30. Jamsheer A, Henggeler C, Wierzba J, Loeys B, De Paepe A, Stheneur Ch, et al. A new sporadic case of early-onset Loeys-Dietz syndrome due to the recurrent mutation $p$. R528C in the TGFBR2 gene substantiates interindividual clinical variability. J Appl Genet. 2009;50:405-10.

31. Tunçbilek E, Alanay Y. Congenital contractural arachnodactyly (Beals syndrome). Orphanet Journal of Rare Diseases. 2006;1:20.

32. Robinson PN, Neumann LM, Demuth S, Enders H, Jung U, König R et al. Shprintzen-Goldberg syndrome: fourteen new patients and a clinical analysis. Am J Med Genet A. 2005;135:251-62.
33. Shprintzen RJ, Goldberg RB. A recurrent pattern syndrome of craniosynostosis associated with arachnodactyly and abdominal hernias. J Craniofac Genet Dev Biol. 1982;2:65-74.

34. Bergman R, Nevet MJ, Gescheidt-Shoshany H, Pimienta AL, Reinstein E. Atrophic skin patches with abnormal elastic fibers as a presenting sign of the MASS phenotype associated with mutation in the fibrillin 1 gene. JAMA Dermatol. 2014;150:885-9.

35. Lang RM, Badano LP, Mor-Avi V, Afilalo J, Armstrong A, Ernande L, et al. Recommendations for cardiac chamber quantification by echocardiography in adults: an update from the American Society of Echocardiography and the European Association of Cardiovascular Imaging. Eur Heart J Cardiovasc Imaging. 2015;16:233-70.

36. McLaughlin VV, Archer SL, Badesch DB, Barst RJ, Farber HW, Lindner JR, et al. American College of Cardiology Foundation Task Force on Expert Consensus Documents; American Heart Association; American College of Chest Physicians; American Thoracic Society, Inc; Pulmonary Hypertension Association. ACCF/AHA 2009 expert consensus document on pulmonary hypertension: a report of the American College of Cardiology Foundation Task Force on Expert Consensus Documents and the American Heart Association developed in collaboration with the American College of Chest Physicians; American Thoracic Society, Inc.; and the Pulmonary Hypertension Association. J Am Coll Cardiol. 2009;53:1573-619.

37. Daunt SW. Cohen JH, Miller SF. Age related normal ranges for the Haller Index in children. Pediatr Radiol. 2004;34:326-30.

38. Williams AM, Crabbe DC. Pectus deformities of the anterior chest wall. Paediatr Respir Rev. 2003:4:237-42.

39. Tocchioni F, Ghionzoli M, Pepe G, Messineo A. Pectus excavatum and MASS phenotype: an unknown association. J Laparoendosc Adv Surg Tech A. 2012:22:508-13.

40. Cartoski MJ, Nuss D, Goretsky MJ, Proud VK, Croitoru DP, Gustin T, et al. Classification of the dysmorphology of pectus excavatum. J Pediatr Surg. 2006;41:1573-81.

41. Rattan AS, Laor T, Ryckman FC, Brody AS. Pectus excavatum imaging: enough but not too much. Pediatr Radiol. 2012;40:168-72.

42. Van Tongerloo A, De Paepe A. Psychosocial adaptation in adolescents and young adults with Marfan syndrome: an exploratory study. J Med Genet. 1998:35:405

43. Wang B, Cao XB, Qiu Y, Qian BP, Sun X, Huang AB, et al. [Pulmonary dysfunction patterns in patients with Marfan and Marfanoid syndrome associated with scoliosis and the influencing factors]. Zhonghua Wai Ke Za Zhi. 2010;48:686-9.

44. Chung JH, Park HJ, Kim KT. Scoliosis after pectus excavatum correction: does it improve or worsen? Eur J Cardiothorac Surg. 2017:52:76-82.

45. Hall JR, Pyeritz RE, Dudgeon DL, Haller JA Jr. Pneumothorax in the Marfan syndrome: prevalence and therapy. Ann Thorac Surg. 1984;37:500-8.

46. Sharma BK, Talukdar B, Kapoor R. Cystic lung in Marfan's syndrome. Thorax. 1989;44:978-9.

47. Sensenig DM, LaMarche P. Marfan's syndrome and spontaneous pneumothorax. Am J Surg. 1980;139:602-4.

48. Landman S, Burgener FA. Pneumothorax in Marfan's disease. N Y State J Med. 1976;76:2180-1.

49. Breslin ABX. Recurrent pneumothorax in a patient with the Marfan syndrome. Med J Aust. 1973;1:1191-3.

50. Chan Wah Hak YS, Lim YP, Liew R, Hsu LF. Pectus excavatum: uncommon electrical abnormalities caused by extrinsic right ventricular compression. J Cardiovasc Electrophysiol. 2014;25:324-7.

51. Dore Reyes M, De La Torre $C$, Bret Zurita M, Triana Junco P, Jiménez Gómez $\mathrm{J}$, Romo Muñoz M, et al. Benefits of magnetic resonance for the study of pectus excavatum in children: initial experience. Cir Pediatr. 2017;30:71-6.

52. Sogawa $M$, Ohzeki $\mathrm{H}$, Namura $\mathrm{O}$, Hayashi J. Preoperative respiratory physiotherapy for a patient with severe respiratory dysfunction and annuloaortic ectasia. Ann Thorac Cardiovasc Surg. 2003;9:266-9.

53. Scherer LR, Arn PH, Dressel DA, Pyeritz RM, Haller JA Jr. Surgical management of children and young adults with Marfan syndrome and pectus excavatum. J Pediatr Surg. 1988;23:1169-72. 